\title{
Search for ultra high energy cosmic ray sources
}

\author{
A.A. Mikhailov ${ }^{1, a}$ and V.A. Kolosov ${ }^{1}$ \\ Yu.G. Shafer Institute of Cosmophysical Research and Aeronomy, 31 Lenin Ave., 677980 Yakutsk, Russia
}

\begin{abstract}
Earlier we suggested a new method to search for sources and anisotropy of the arrival directions of ultra high energy particles. Here using this method we analyzed the data of the Yakutsk EAS array and found that particle fluxes with energies $E<4.10^{19} \mathrm{eV}$ come to the Earth from our Galaxy and the arrival directions of particles correlate with known pulsars. The chance probability of correlating particles with pulsars and the anisotropy of the distribution of particles is $10^{-4}-10^{-5}$.

We also analyzed the Telescope Array (TA) data with energies $E<4.10^{19} \mathrm{eV}$ by our method and found that the maximum of the distribution of particles points to extragalactic sources but the direction of the maximum does not coincide with the maximum direction of the distribution of particles found by the authors of the Telescope Array data (the maximums of the arrival directions received by two different methods of analyses differ by about $10 \%)$.
\end{abstract}

\section{Introduction}

From 1974 near Yakutsk, Russia, an extensive air shower array (EAS) of area $18 \mathrm{~km}^{2}$ began to register extensive air showers. The Yakutsk array is situated in North Asia near the North Ice ocean at a distance of $50 \mathrm{~km}$ south-west from the city of Yakutsk in the Lena river valley near the village of Oktyomsy. The geographical coordinates are: latitude $61.7^{\circ} \mathrm{N}$ and longitude $129.4^{\circ} \mathrm{E}$ at a height of $100 \mathrm{~m}$ above sea level, i.e., 1020 g.cm².

The Yakutsk array consists of 49 surface scintillation plastic detectors each of area $2 \times 2 \mathrm{~m}^{2}, 9$ surface scintillation plastic detectors of area $2 \mathrm{~m}^{2}$ for electrons and photons, 5 underground scintillation plastic detectors of $20 \mathrm{~m}^{2}$ for muons with an energy threshold of $E_{\mu}>1 \times$ $\sec \theta \mathrm{GeV}$ (where $\theta$ is the zenith angle) and 45 Cherenkov light detectors for registering electrons and photons. The primary particle energy is estimated by a calorimetric method using the Cherenkov light of an EAS. Since 1990 the surface detectors formed a uniform net of $18 \mathrm{~km}^{2}$ area (until 1990 the array area was $10 \mathrm{~km}^{2}$ ) with a distance of $500 \mathrm{~m}$ between neighboring units. In the center of the array is a muon detector of $192 \mathrm{~m}^{2}$ to register muons with energy $E_{\mu}>0.5 \times \sec \theta \mathrm{GeV}$. Timing signals for each detector are distributed over a microwave link: a resolution of $10^{-7} \mathrm{~s}$ is achieved. A full description of the array has been given in references $[1,2]$.

We analyzed the distribution of particles in the celestial sphere of the Yakutsk array data [3] by classic methods (harmonics analyses) and by our suggested method. We considered the arrival directions of extensive air shower for particles with zenith angles $\theta<60^{\circ}$ and cores located inside the array perimeter. The threshold energy of the primary particles was $E \geq 5.10^{18} \mathrm{eV}$ and the energy and arrival direction were determined with an accuracy of $\sim 30 \%$ and $\sim 5-7^{\circ}$ respectively.

a e-mail: mikhailov@ikfia.ysn.ru

\section{The distribution of particles in right ascension}

We considered the Yakutsk array data from 1974 until 2011 for energies $E \geq 5.10^{18} \mathrm{eV}$. At first we analysed the arrival distribution of particles in the celestial sphere by harmonic analysis.

Figures 1 and 2 show the distribution of particles in right ascension detected by the Yakutsk array for the period 1974-2011 for different energy intervals, where the shower axea are situated inside the perimeter of the array:

- for the energy interval, $E=(5-8) 10^{18} \mathrm{eV}$ (Fig. 1), the amplitudes and phases in right ascension of the first harmonic of Fourier analysis are: first harmonic, $r_{1}$, and phase, $\phi_{1}$, of the maximum: $r_{1}=0.037 \pm 0.04$, $\phi_{1}=76^{\circ} \pm 58^{\circ}$, second harmonics are $r_{2}=0.04 \pm$ $0.04, \phi_{2}=42^{\circ} \pm 48^{\circ}$, number of particle $n=1469$. The chance probability, $\mathrm{P}$, to have amplitudes $r_{1} \geq 0.037$ according to Linsley [4] is $\mathrm{P} \sim 0.6$;

- for energies $E \geq 8.10^{18} \mathrm{eV}$ (Fig. 2), the amplitude and phase in right ascension of the $1^{\text {st }}$ harmonic of Fourier analysis are $r_{1}=0.14 \pm 0.04, \phi_{1}=0.6^{\circ} \pm 19^{\circ}$, the number of particles $\mathrm{n}=938$; the chance probability, P, to have amplitudes $r_{1} \geq 0.14$ is $P \sim 0.01$.

In our opinion, the method of analysing by harmonic functions is not effective because of the results of summing the number of particles in declination (this summing is demanded by the method), and some local maximums and minimums in declination can smooth them out. Because of the smooth data, a received anisotropy of particle arrival directions perhaps differs from reality. In addition, this method only analyzes data along one coordinate - Right Ascension. Therefore, this analysis can only be used for a preliminary data analysis. Harmonic analyses point to galactic origin (Table 1).

We therefore suggested a new method $[5,6]$ which analyzes data using two coordinates: declination and right ascension. Using this method we analyzed the extensive 
Table 1. Amplitudes and phases of the analysis of particle distributions in Right Ascension.

\begin{tabular}{|c|c|c|c|c|c|c|}
\hline $\begin{array}{c}\text { Energy } \\
\text { ev }\end{array}$ & $\begin{array}{c}\mathrm{N} \\
\text { Number } \\
\text { of particles }\end{array}$ & $\begin{array}{c}\mathrm{A}_{1} \% \\
1^{\text {st }} \text { harm. }\end{array}$ & $\begin{array}{c}\mathrm{A}_{2} \% \\
2^{\text {nd }} \text { harm. }\end{array}$ & $\begin{array}{c}\phi_{1} \\
\text { deg. }\end{array}$ & $\begin{array}{c}\phi_{2} \\
\text { deg. }\end{array}$ & $\begin{array}{c}\mathrm{P} \\
\text { chance } \\
\text { probability }\end{array}$ \\
\hline$(5-8) 10^{18}$ & 1469 & 3.6 & 4.4 & $76^{\circ}$ & $42^{\circ}$ & 0.6 \\
\hline$(8-40) 10^{18}$ & 938 & 0.14 & 0.02 & $7^{\circ}$ & $7.6^{\circ}$ & 0.012 \\
\hline
\end{tabular}

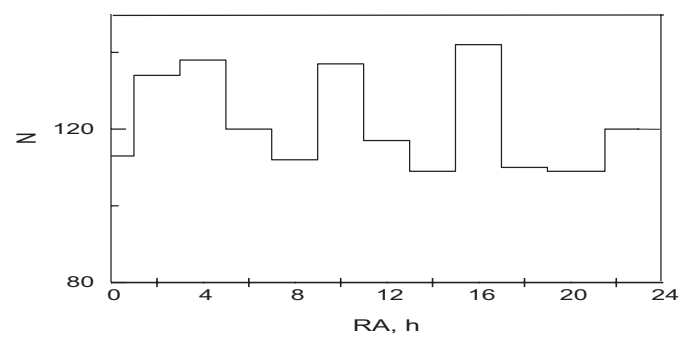

Figure 1. The distribution of particles in right ascension with energies $\mathrm{E}=(5 \div 8) 10^{18} \mathrm{eV}$; Number of particles $\mathrm{N}=1469$.

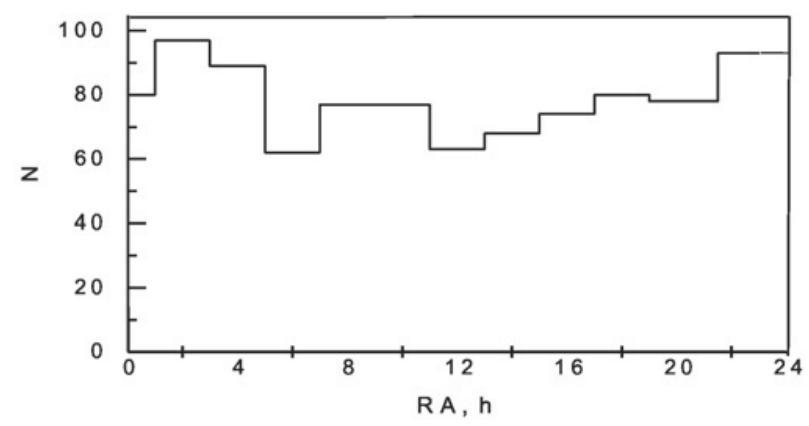

Figure 2. The distribution of particles in right ascension with energies $E \geq 8.10^{18} \mathrm{eV}$; Number of particles $\mathrm{N}=938$.

air shower data of Yakutsk and the Telescope Array. We considered the Yakutsk data registered for 1974-2011 with shower axes inside the perimeter of the array. As a result of our analyses we concluded [1,7-15] that cosmic rays up to energy $E \sim 4.10^{19} \mathrm{eV}$ are galactic and their sources are pulsars [16].

\section{The particle flux from the galactic plane}

We consider the distribution of particles with energies $E \geq 8.10^{18} \mathrm{eV}$ in galactic latitude, b, in $3^{\circ}$ bins. The distribution of particles in galactic latitude is shown in Fig. 4; the expected numbers of events at the observation level is the dashed line. Note that the expected numbers of events have been determined by simulating $10^{6}$ random events using the Monte-Carlo method. Events are simulated in the horizontal coordinate system taking into account the zenith-azimuth distribution of particles $d n(\theta) \sim \cos (\theta) \sin (\theta) d \theta$ and in azimuth angle $\phi$ uniformly. Then, the distribution of the events was transferred into the galactic system coordinates.

As seen in Fig. 4, at latitudes $b \sim 0^{\circ}$ an excess is seen of the observed number of showers compared with those expected. In the latitude interval $0 \leq b<3^{\circ}$ the excess of the number of showers is

$$
\left(n_{o b s}-n_{\text {exp }}\right) / \delta n_{\text {exp }} \text { is } \sim 3.5 \sigma
$$
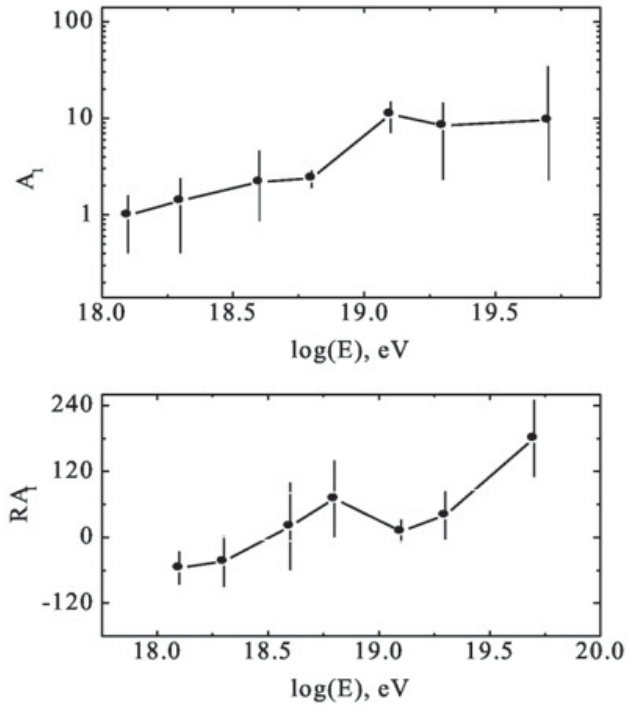

Figure 3. Amplitudes and phases of the $1^{\text {st }}$ harmonic in the shower distribution depending on their energy.

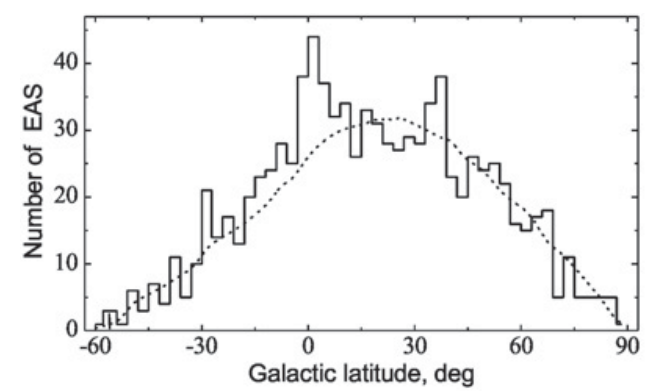

Figure 4. The distribution of particles with energy $\mathrm{E} \geq 8.10^{18} \mathrm{eV}$ in galactic latitude

where the observed number of showers $n_{\mathrm{obs}}=45$. This distribution of phases, first harmonics maximum of particles indicates that, most likely, particles up to $\sim 10^{19} \mathrm{eV}$ are galactic in origin. There are also two excesses of particles at latitudes $-30^{\circ} \leq b \leq-27^{\circ}$ and $33^{\circ} \leq b \leq 39^{\circ}$.

Results of harmonic analyses and the distribution of particles in galactic latitude also indicate that particles up to $4.10^{19} \mathrm{eV}$ are galactic in origin.

\section{Method to search for sources and anisotropy}

We suggest a new method to search for sources and anisotropy of the arrival directions of ultrahigh energy cosmic rays [6]: around possible sources (pulsars) we drew circles with an angular distance, $\mathrm{R}$, equal to or greater than the deviation angles of particles from the sources to Earth. Then we find the number of particles inside these circles and choose those circles as sources where the number of particles inside the circles, $<\mathrm{R}$, is above a selected number of particles, $>\mathrm{n}$, (usually $\mathrm{n}$ is the mean number of particles).

If the circles around proposed sources overlap, then we unite them into one region. Furthermore we determine the chance probability, $\mathrm{P}$, to observe a number of particles, $\mathrm{n}_{1}$, in the considered region of the celestial sphere. In our initial study we found a correlation of particle 


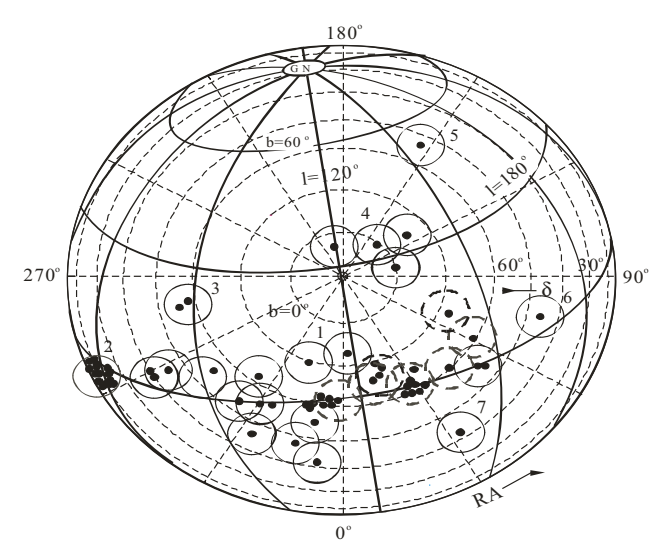

Figure 5. Yakutsk, $E \geq 8.10^{18} \mathrm{eV}$, correlation of particles with pulsars, circles around pulsars at $\mathrm{R}=6^{\circ}$ and number of particles inside circles $n \geq 10$.

arrival directions with pulsars, suggested as most probable sources of particles, by our analysis data at angular radius $\leq 6^{\circ}$ for energies $E \geq 10^{19} \mathrm{eV}$ for the Yakutsk EAS data [7]. We therefore take this angular distribution as a pattern for the correlation between particles and galactic sources, pulsars.

\section{Correlation of particles with pulsars}

From 1974 to 2011 the Yakutsk EAS array registered 938 particles for $\mathrm{E}>8.10^{18} \mathrm{eV}$ inside the perimeter of the array. We select those pulsars [16] as sources of cosmic rays if the number of particles inside the radius $\mathrm{R}<6^{\circ}$ from the considered pulsar was $\mathrm{n} \geq 10$. If circles around pulsars overlap then we unite them into one region. The distribution of particles is shown on the map of equal exposure of the celestial sphere where an equal number of particles is expected from equal regions [1]. We have found 7 regions: inside the $1^{\text {st }}$ region (Fig. 5) -39 pulsars, inside the $2^{\text {nd }}$ region -16 pulsars, the $3^{\text {rd }}$ region-2 pulsars, the $4^{\text {th }}$ region-4 pulsars and separately pulsars 5-7 (Fig. 6) and inside these regions $183,17,11,33,14,11,10$ particles respectively. The chance probabilities to find such number of events from 938 particles are: $P_{1}(938 \geq 183) \sim 6.10^{-5}, \quad P_{2}(938 \geq$ 17) $\sim 1.8 \times 10^{-3}, P_{3}(938 \geq 11), \sim 0.6, P_{4}(938 \geq 33) \sim$ $0.5, P_{5}(938 \geq 11) \sim 0.6, P_{6}(938 \geq 33) \sim 0.5, P_{7}(938 \geq$ 14) $\sim 0.04, P_{6}(938 \geq 11) \sim 0.1$ and $P_{7}(938 \geq 10) \sim 0.1$.

Note that chance probabilities are only significant around the $1^{\text {st }}$ and $2^{\text {nd }}$ groups of pulsars (Fig. 6) at radius $\mathrm{R}_{i} 6^{\circ}$ and are $3 \sigma$ more than expected for the case of isotropy. The maximum number of events $20 \leq n \leq 23$ at $\mathrm{R}<6^{\circ}$ are observed around the $1^{\text {st }}$ groups of pulsars: PSR $0141+6009,0146+6145,0147+5922,0157+$ $6212,0215+6218$. These pulsars are in the galactic plane and have been considered earlier as possible sources of particles [1].

A flux of particles was observed from the side center of the Galaxy (Fig. 5, number 2). All 14 particles arrive at large zenith angles $60^{\circ} \geq \theta \geq 38^{\circ}$.

We considered the Telescope array EAS data for energies $E \geq 5.7 \times 10^{19} \mathrm{eV}$ [3] and found 31 particles at $\mathrm{R}<6^{\circ}$ from pulsars. The chance probability to find such a number of particles from 72 is $P \sim 0.1$. Thus we did not

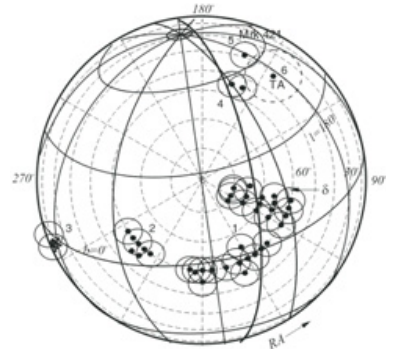

Figure 6. Yakutsk, $E \geq 8.10^{18} \mathrm{eV}$, anisotropy: distribution of points in the celestial sphere $\bullet$ within which at radius $\mathrm{R}<6^{\circ}-$ $\geq 16$ particles were observed. 1, 2, 3, 4-data of Yakutsk array. 5-circles around the galaxy Mkr 421, 6-TA-maximum particles found by the Telescope Array data, $\mathrm{R}<20^{\circ}$.

Table 2. The energy and co-ordinates of particles which came from the Galactic Center during 1974-2014.

\begin{tabular}{|c|c|c|c|}
\hline $\begin{array}{c}\text { Energy } \\
\text { ev }\end{array}$ & $\begin{array}{c}\mathrm{N} \\
\text { Number } \\
\text { of particles }\end{array}$ & $\delta$ & RA \\
\hline$>4 \cdot 10^{19}$ & 14 & $\sim 20^{\circ}$ & $290^{\circ}$ \\
\hline
\end{tabular}

find any correlation with pulsars in the arrival directions of particles from the TA array data.

\section{Anisotropy of ultrahigh energy particles}

We have estimated the anisotropy of the arrival directions of ultrahigh energy particles. According to our method $[5,6]$ it draws lines of a coordinate grid at declination, $\delta$, and right ascension, RA, on the celestial sphere through $5^{\circ} \times 5^{\circ}$, beginning with declination $\delta=0^{\circ}$ and right ascension $\mathrm{RA}=0^{\circ}$. We have counted up the number of observed particles at $\mathrm{R}<6^{\circ}$ around points where the above-stated coordinates are crossed.

We selected those points around which at $\mathrm{R}<6^{\circ}$ the observed number of particles was $n \geq 16$ events for the Yakutsk array data (Fig. 7). We found in the $1^{\text {st }}$ region 115 particles after the connection of regions $\mathrm{R}<6^{\circ}$ which form as a result of cross declinations and right ascensions $5^{\circ} \times 5^{\circ}$; in the $2^{\text {nd }}$ region-24 particles, in $3^{\text {rd }}$ region-14 particles, in $4^{\text {th }}$ region-32 particles. The probabilities to find such a number of events from 938 particles in these regions are: $1-P(938 \geq$ $115) \sim 2.10^{-5}, \quad 2-P(938 \geq 24) \sim 10^{-2}, \quad 3-P(938 \geq$ 14) $\sim 3.10^{-4}, 4-P(938 \geq 32) \sim 8.10^{-2}$.

It is seen in Fig. 6 that the maximum of the distribution of particles is around the coordinates: $\delta=60^{\circ}$, RA $=$ $25^{\circ}-40^{\circ}$ (number of particles in this region for $\mathrm{R}<$ $6^{\circ}, n \geq 18$ ). This maximum of the particle distribution coincides with the position of the following pulsars: PSR $0141+6009,0146+6145,0147+5922,0157+6212$, $0215+6218) .23$ particles were observed at $\mathrm{R}<6^{\circ}$ around the peak of this maximum at $\delta=60^{\circ}$ and $\mathrm{RA}=25^{\circ}$ and the chance probability is $P(938, \geq 23) \sim 2.10^{-4}$.

The number of particles in the Yakutsk data is not enough to make any conclusion (at energy $E>4.10^{19} \mathrm{eV}$ there were $<50$ particles).

In addition we also analyzed the TA data. We did not find any excess of particles in the TA data around the galaxy Mkr 421 (Fig. 7) (our colleagues [3] found 


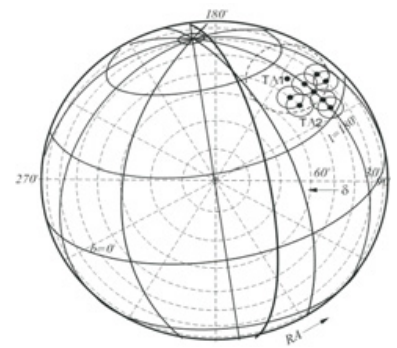

Figure 7. Telescope array data, $E \geq 4.10^{19} \mathrm{eV}$, TA1-maximum distribution of particles found by the authors of the Telescope Array data, $\mathrm{R}<20^{\circ}$. Around 8 points at $\mathrm{R}<6^{\circ}$ maximum distribution of particles by our method, $\mathrm{n} \geq 3$ particles.

an excess of the particle flux from this galaxy). Only 5 particles at $\mathrm{R}<6^{\circ}$ around this galaxy from 72 particles were observed and the chance probability by our method is only $\mathrm{P} \sim 0.6$.

According to the TA data 19 particles were observed at a radius $\mathrm{R}<20^{\circ}$ (dashed curve around the TA hotspot, Fig. 4) centered around the coordinate $\delta=43.2^{\circ}$, RA $146.7^{\circ} \quad$ [3]. We estimate the chance probability using the TA data [3] and our method is only $\mathrm{P} \sim 10^{-2}$. Our method differs from the method used by the authors of the TA collaboration.

We therefore decided to search for any anisotropy in the TA data using our method. Curved lines were drawn on a coordinate grid $5^{\circ} \times 5^{\circ}$ around the whole celestial sphere and we selected those points where coordinates crossed and demanded that the number of observed particles was $n \geq 3$ from these points at distances $\mathrm{R}<6^{\circ}$. Such a number of particles was observed around only 8 points at declinations $\delta=40^{\circ}, 50^{\circ}, 55^{\circ}$ and right ascensions $125^{\circ}$, $130^{\circ}, 135^{\circ}, 140^{\circ}$ crossing (Fig. 3,8 points, TA2). Around these 8 points at radius $\mathrm{R}<6^{\circ}$ there are 8 particles and the chance probability, $\mathrm{P}$, to observe 8 particles from 72 by our method [5] is $P(72, \geq 8) \sim 3.10^{-5}$.

\section{Conclusion}

We have found correlations in the arrival directions of ultrahigh energy particles with $E<4.10^{19} \mathrm{eV}$ with the positions of pulsars and an anisotropy of the arrival directions of these particles from the side of the galactic plane in the Yakutsk array data and the Telescope Array data at energy $E>5.7 \cdot 10^{19} \mathrm{eV}$ at high galactic latitudes. Our analysis of the data shows that the cosmic rays observed up to $4 \cdot 10^{19} \mathrm{eV}$ are galactic and above this energy most likely extragalactic.

\section{References}

[1] Dyakonov M.N., Efimov N.N., Egorov T.A., Mikhailov A.A., et al. Kozmicheskoe izluchenie predelno visokoi energii. Novosibisk, Monograph, Nauka, 1991, 251

[2] Afanasiev B.N., Glushkov A.V., Egorova V.P, Mikhailov A.A. et al., Some Characteristics of EAS and Primary Cosmic Rays on Yakutsk Array data. In: Proceedings of the Tokyo Workshop on Techniques for the Study of the Extremely High Energy Cosmic Rays, edited by M. Nagano, Tokyo, 1996, 32

[3] Abbasi R.U., Abe M., T. Abu-Zayad et al., 2014: Arxiv: 1404.5890v3

[4] Linsley J., Phis. Rev. Lett., 1975, 34, 1530-1533

[5] Efimov N.N., Mikhailov A.A. Astropart. Phys. 1994, 2, 329

[6] Mikhailov A.A., Analysis of the Arrival Directions of Ultrahigh Energy Particles. Discovery of Cosmic Rays, book, Denver, June 26-28, 2012, ed. J.Ormes

[7] Mikhailov A.A., Izvestia RAN, 1999, ser.fiz. 63, 556

[8] Mikhailov A.A., Astronom. J. 1979, 56, 505

[9] Mikhailov A.A., Syrovatsky S.I. Pisma v AJ, 1979, 56, 505

[10] Berezinsky V.S., Mikhailov A.A. Pisma v JETF, 1984, 10(4), p. 269

[11] Berezinsky V.S., Mikhailov A.A. Pisma v AJ, 1984, 10(4), p. 269

[12] Erlykin A.D., Mikhailov A.A., Wolfendale A.W., J. Phys. G., Nucl, Part. Phys., 2002, 8

[13] Becker P.A., G.S., Bisnovatyi-Kogan, D., Casadei, D., Mikhailov A.A. et al., Frontiers in Cosmic Rays Research, Monograph, NY, Nova Science Publishers, Inc. NY, 2010, 161

[14] Malov I.E., Mikhailov A.A.,Avramenko A., Lorenzo Iorio et al., Pulsars: Theory, Categories and Application., Monograph, 2011, Nova Science Publishers, Inc. NY, 31

[15] Dorman L.I., Dorman I.V., Ahluwalia H.S., Mikhailov A.A. et al., Homage the Discovery of Cosmic Rays, the Meson-Muon and Solar Cosmic Rays. Monograph, New York, Nova Science Publishers, Inc. NY, 301

[16] Manchester R.N., Hobbs G.B., Teoh A., \& Hobbs M., AJ., 2005, 129, 1993

[17] V.A.Kuzmin, V.A. Rubakov, Yadernaya Fizika, 1998, 61, 1122

[18] Anda R., Aguirre C., Tachi K., Proc.17-th ICRC, Paris, 1981, 2, 164

[19] Bray A.D., Horton L., McCusker C.B.A. et al., Proc. 17-th ICRC, 1981, 2, 168 